\title{
Gallstone ileus: Report of two cases and review of the literature
}

\author{
Jen-Wei Chou, Chang-Hu Hsu, Kuan-Fu Liao, Hsueh-Chou Lai, Ken-Sheng Cheng, Cheng-Yuan Peng, \\ Mei-Due Yang, Yung-Fang Chen
}

Jen-Wei Chou, Chang-Hu Hsu, Kuan-Fu Liao, HsuehChou Lai, Ken-Sheng Cheng, Cheng-Yuan Peng, Division of Gastroenterology, Department of Internal Medicine, China Medical University Hospital, Taichung 40447, Taiwan, China

Mei-Due Yang, Department of Surgery, China Medical University Hospital, Taichung 40447, Taiwan, China

Yung-Fang Chen, Department of Radiology, China Medical University Hospital, Taichung 40447, Taiwan, China

Correspondence to: Dr. Hsueh-Chou Lai, MD, Division of Gastroenterology, Department of Internal Medicine, China Medical University Hospital, No.2, Yuh-Der Road, North District, Taichung 40447, Taiwan,

China. adenocarcinomalai@yahoo.com.tw

Telephone: +886-4-22052121-2220 Fax: +886-4-22023119

Received: 2006-11-08

Accepted: 2007-02-12

\begin{abstract}
Gallstone ileus is a rare disease and accounts for $1 \%-4 \%$ of all cases of mechanical intestinal obstruction. It usually occurs in the elderly with a female predominance and may result in a high mortality rate. Its diagnosis is difficult and early diagnosis could reduce the mortality. Surgery remains the mainstay of treatment. We report two cases of gallstone ileus. The first was a 78-year old woman who had a 2-d history of vomiting and epigastralgia. Plain abdominal film suggested small bowel obstruction clinically attributed to adhesions. Later on, gallstone ileus was diagnosed by abdominal computed tomography (CT) based on the presence of pneumobilia, bowel obstruction, and an ectopic stone within the jejunum. She underwent emergent laparotomy with a one-stage procedure of enterolithotomy, cholecystectomy and fistula repair. The second case was a 76-year old man with a 1-wk history of epigastralgia. Plain abdominal film showed two round calcified stones in the right upper quadrant. Fistulography confirmed the presence of a cholecystoduodenal fistula and gallstone ileus was also diagnosed by abdominal CT. We attempted to remove the stones endoscopically, but failed leading to an emergent laparotomy and the same one-stage procedure as for the first case. The postoperative courses of the two cases were uneventful. Inspired by these 2 cases we reviewed the literature on the cause, diagnosis and treatment of gallstone ileus.
\end{abstract}

(C) 2007 The WJG Press. All rights reserved.

Key words: Gallstone ileus; Intestinal obstruction; Pneumobilia; One-stage procedure; Enterolithotomy;
Cholecystoduodenal fistula

Chou JW, Hsu CH, Liao KF, Lai HC, Cheng KS, Peng CY, Yang MD, Chen YF. Gallstone ileus: Report of two cases and review the literature. World J Gastroenterol 2007; 13(8): 1295-1298

http://www.wjgnet.com/1007-9327/13/1295.asp

\section{INTRODUCTION}

The cause of mechanical small bowel intestinal obstruction includes gallstones, foreign bodies, bezoars, tumors, adhesions, congenital abnormity, intussusceptions, and volvulus ${ }^{[1]}$. Among these causes, a gallstone-induced intestinal obstruction is also referred to as a "gallstone ileus". Gallstone ileus is a rare and potentially serious complication of cholelithiasis ${ }^{[2]}$. It accounts for $1 \%-4 \%$ of all cases of mechanical intestinal obstruction, but for up to $25 \%$ of those in patients over 65 years of age with a female to male ratio of $3.5-6.0: 1^{[3-6]}$. The morbidity and mortality rate of gallstone ileus remain very high, partly because of misdiagnosis and delayed diagnosis ${ }^{[7]}$. Thus, early diagnosis and prompt treatment could reduce the mortality rate. Here, we report two cases of gallstone ileus and review the literature of this rare disease.

\section{CASE REPORTS}

\section{Case 1}

A 78-year old woman presented to our emergency department (ED) with a 2-d history of vomiting and epigastric pain. She had a past medical history of hypertension and underwent a hystectomy for a myoma at the age of 38 . On physical examination, her abdomen was mildly distended without tenderness or rebound or Murphy's sign. The white blood count was $16630 / \mu \mathrm{L}$ with $87.6 \%$ neutrophils, blood urea nitrogen $67 \mathrm{mg} / \mathrm{dL}$, $2.3 \mathrm{mg} / \mathrm{dL}$ creatinine $2.3 \mathrm{mg} / \mathrm{dL}$ and $\mathrm{k} 2.7 \mathrm{mmol} / \mathrm{L}$. The other tests were unremarkable. A plain abdominal film demonstrated mildly dilated bowel loops, but a vague stone was not identified in the left iliac fossa (Figure 1). The diagnostic impression was ileus due to adhesions. Abdominal ultrasound (US) disclosed a shrunken gallbladder with internal air, but without stones. An abdominal computed tomography was performed, demonstrating air in the biliary tree (Figure 2A). Moreover, 


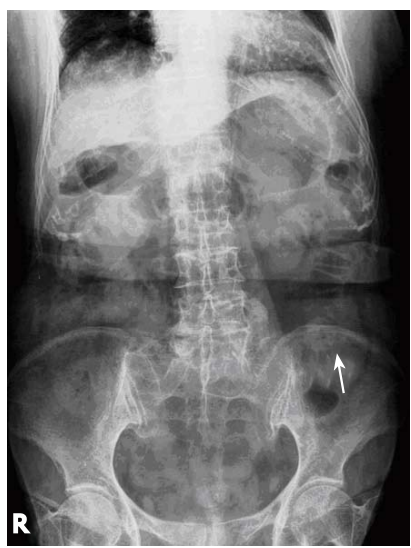

Figure 1 Plain abdominal film showing a vague stone not identified in the left iliac fossa (arrow).

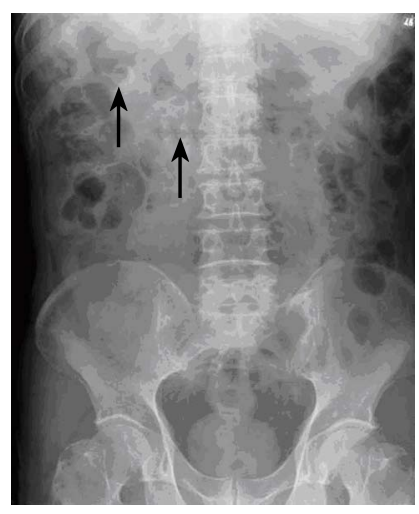

Figure 3 Plain abdominal film showing two round calcified stones in the right upper quadrant of abdomen (arrow).

an ectopic stone impacted in the jejunal lumen was seen in the left lower quadrant along with dilatation of the proximal small bowel (Figure $2 \mathrm{~B}$ ). The diagnosis of gallstone ileus was made and she underwent an emergent laparotomy. At operation a small shrunken gallbladder with dense adhesion to the stomach was found. After lysis of adhesion, a fistula between the gallbladder dome and the lesser curvature of gastric antrum was confirmed. Moreover, a stone was palpated in the jejunum about $140 \mathrm{~cm}$ distal to the ligament of Treitz causing total obstruction of the lumen. When the jejunum was opened, the stone was laminated and cylinder shaped, measuring $3 \mathrm{~cm} \times 3 \mathrm{~cm} \times 2 \mathrm{~cm}$ in diameter. Enterolithotomy, cholecystectomy and repair of the cholecystogastric fistula were performed. The pathology showed chronic cholecystitis and a fistula walled by granulation tissue. The postoperative course was uneventful except for mild wound infection, and she remained in good health after one-year follow-up.

\section{Case 2}

A 76-year old man was brought to our ED because of epigastric pain for one week. He denied any history of abdominal surgery or biliary disease. On physical examination his abdomen was soft, but tender in the epigastric area. Laboratory tests were unremarkable. Abdominal X-ray showed two round calcified stones in the right upper quadrant of the abdomen (Figure 3). An EGD disclosed a deep hole in the posterior wall of the duodenal bulb, but no stone was identified. Fistulography confirmed the presence of a cholecystoduodenal fistula.
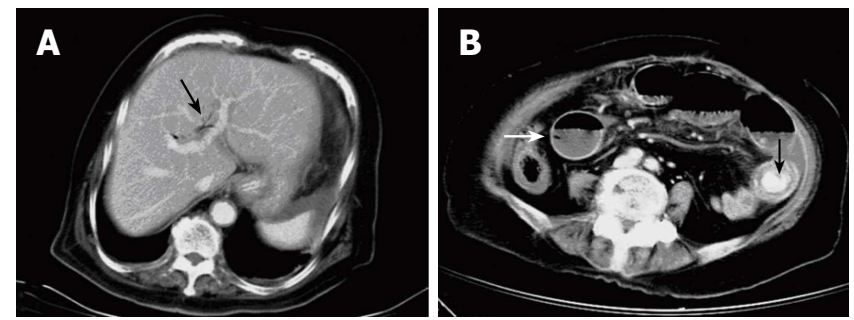

Figure 2 Abdominal computed tomography scan showing air in the biliary tree (arrow) (A) and an ectopic stone (arrow) impacting the jejunal lumen accompanying dilatation of proximal small bowel loops (white arrow) (B).
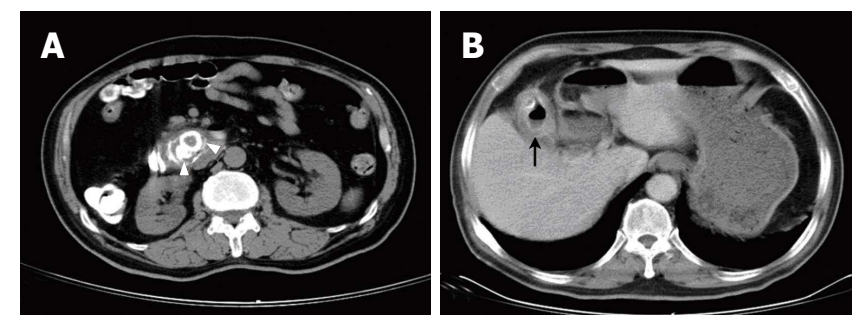

Figure 4 Abdominal computed tomography scan showing two round calcified stones impacting the proximal third portion of the duodenum (arrowhead) (A) and a thickened wall gallbladder with internal air and small calcified content (arrow) (B).

An abdominal CT was performed, showing two round calcified stones located in the proximal third portion of the duodenum causing dilatation of proximal bowel lumen (Figure 4A). There was a thickened wall gallbladder with internal gas and small calcified content was also discovered (Figure 4B). Duodenoscopy was performed again, showing two stones impacted in the proximal third portion of the duodenum. We attempted to catch the stones with a Dormia basket, but failed because of their larger size. Therefore, he was referred to our department of surgery for emergent laparotomy. Laparotomy displayed that the gallbladder was adhered to the duodenum accompanying a cholecystoduodenal fistula. A large black stone, measuring $5 \mathrm{~cm} \times 4 \mathrm{~cm} \times 3 \mathrm{~cm}$ in diameter, impacted the proximal third portion of the duodenum. No other stones were found in the gastrointestinal (GI) tract. Enterolithotomy, cholecystectomy, duodenorrhaphy, feeding jejunostomy, and repair of the cholecystoduodenal fistula were performed. Microscopically, the gallbladder showed cholelithiasis with acute and chronic inflammation and a fistula walled by fibrous and granulation tissue. The postoperative course was complicated by a wound infection, and he was discharged 2 mo later.

\section{DISCUSSION}

Gallstone is a common disease with a prevalence in $10 \%$ of the adult population in the United States ${ }^{[8]}$. However, it is only symptomatic in $20 \%-30 \%$ of patients, with biliary colic being the most common symptom ${ }^{[9]}$. The most common complications of gallstone disease include acute cholecystitis, acute pancreatitis, choledocholithiasis with or without cholangitis, and a gangrenous gallbladder. Other uncommon complications include Mirizzi syndrome, 
cholecystocholedochal fistula, and gallstone ileus ${ }^{[2,10]}$.

The term "gallstone ileus" was first coined by Bartolin in 1654 and referred to the mechanical intestinal obstruction due to impaction of one or more large gallstones within the GI tract. Biliary-enteric fistula is the major pathologic mechanism of gallstone ileus ${ }^{[11]}$. The gallstone enters the GI tract through a fistula between a gangrenous gallbladder and the GI tract. Occasionally a stone may enter the intestine through a fistulous communication between the common bile duct and the GI tract. Although the gallstone can impact anywhere in the GI tract, its size should be at least $2 \mathrm{~cm}$ to $2.5 \mathrm{~cm}$ in diameter to cause obstruction ${ }^{[6]}$. Reisner and Cohen ${ }^{[5]}$ reviewed 1001 cases of gallstone ileus and reported that the most common locations of impaction of gallstone are the terminal ileum and the ileocecal valve because of the anatomical small diameter and less active peristalsis. They also found that the less common locations for impaction are the jejunum, the ligament of Treitz, and the stomach, while the duodenum and colon are the rare locations for impaction $^{[5]}$.

The clinical manifestations of gallstone ileus are variable and usually depend on the site of obstruction. The onset may be manifested as acute, intermittent or chronic episodes $^{[12]}$. The most common symptoms include nausea, vomiting and epigastric pain. Moreover, a small portion of patients may present with hematemesis secondary to duodenal erosions. Laboratory studies may show an obstructive pattern with elevated values of bilirubin and alkaline phosphophatase.

The diagnosis of gallstone ileus is difficult, usually depending on the radiographic findings. In $50 \%$ of cases the diagnosis is often only made at laparotomy ${ }^{[5]}$. The classic Rigler's triad of radiography includes mechanical bowel obstruction, pneumobilia, and an ectopic gallstone within bowel lumen. However, air in the gallbladder is also a frequent finding in gallstone ileus ${ }^{[13]}$. Plain abdominal films usually show non-specific findings because only $10 \%$ of gallstones are sufficiently calcified to be visualized radiographically. For example, in our first case, although the ectopic gallstone appeared on the initial plain abdominal film, it was misdiagnosed as fecal material due to insufficient calcification. But in our second case, two gallstones were definitely identified on a plain abdominal film. Upper or lower GI barium studies occasionally identify the site of obstruction or fistula. Abdominal US is useful to confirm the presence of cholelithiasis and may identify fistula, if present ${ }^{[14]}$. Abdominal CT becomes the more important modality in diagnosing gallstone ileus because of its better resolution. By comparing with plain abdominal film and abdominal US, it can provide a more rapid and specific diagnosis in emergency use. Lassandro et $a t^{[15]}$ compared the clinical value of plain abdominal film, abdominal US and abdominal CT in diagnosing 27 cases of gallstone ileus, and found that the Rigler's triad presents $14.81 \%$ in plain abdominal film, $11.11 \%$ in abdominal US, and $77.78 \%$ in abdominal CT, respectively. Additionally, Yu et a ${ }^{[16]}$ studied the value of abdominal CT in the diagnosis and management of gallstone ileus and concluded that the abdominal CT offers crucial evidence not only for the diagnosis of gallstone ileus but also for decision making in management strategy ${ }^{[16]}$. Rarely, laparoscopy is used to diagnose this disease ${ }^{[17]}$.

Gallstone ileus usually requires emergent surgery to relieve intestinal obstruction. Bowel resection is only indicated when there is intestinal perforation or ischemia ${ }^{[18]}$. There is no uniform surgical procedure for this disease because of its low incidence. Although enterolithotomy alone remains the popular operative method in most reports, the one-stage procedure composed of enterolithotomy, cholecystectomy and repair of fistula is necessary, if indicated ${ }^{[19]}$. Tan et a ${ }^{[20]}$ compared the two surgical strategies of enterolithotomy alone and enterolithotomy with cholecystectomy for the emergent treatment of gallstone ileus, and concluded that both procedures are safe with no mortality, but the better surgical option is enterolithotomy. Doko et al ${ }^{[21]}$ agreed that the one-stage procedure should be reserved only for highly selected patients with absolute indications. Recently, laparoscopy-guided enterolithotomy has become the preferred surgical approach in treating gallstone ileus ${ }^{[22]}$. Additionally, the non-surgical treatment of gallstone ileus has been suggested, including endoscopic removal and shockwave lithotripsy, but this depends on the location of obstruction ${ }^{[23,24]}$. In our second case, the two stones were detected by radiography preoperatively, but they were not found on the first EGD. The second duodenoscopy revealed two stones impacting the third portion of the duodenum. We attempted to remove them endoscopically, but failed due to their larger size.

The prognosis of gallstone ileus is usually poor and worsens with age. Previous studies reported that the mortality rate is $7.5 \%-15 \%{ }^{[5,6]}$, largely due to delayed diagnosis and concomitant conditions such as cardiorespiratory disease, obesity and diabetes mellitus. The postoperative recurrence rate of gallstone ileus is $4.7 \%$ and only $10 \%$ of patients require secondary biliary surgery for recurrent biliary symptoms ${ }^{[5,25]}$.

In conclusion, gallstone ileus is a rare cause of intestinal obstruction. It must be considered in intestinal obstruction patients with a past history of gallstone, especially in elderly females. Abdominal CT is the preferred modality because of its rapid diagnosis of gallstone ileus. Surgical treatment is emergent when the radiological finding is highly or even suspicious or confirmed.

\section{REFERENCES}

1 Richards WO, Williams LF. Obstruction of the large and small intestine. Surg Clin North Am 1988; 68: 355-376

2 Abou-Saif A, Al-Kawas FH. Complications of gallstone disease: Mirizzi syndrome, cholecystocholedochal fistula, and gallstone ileus. Am J Gastroenterol 2002; 97: 249-254

3 Kurtz RJ, Heimann TM, Kurtz AB. Gallstone ileus: a diagnostic problem. Am J Surg 1983; 146: 314-317

4 Clavien PA, Richon J, Burgan S, Rohner A. Gallstone ileus. Br J Surg 1990; 77: 737-742

5 Reisner RM, Cohen JR. Gallstone ileus: a review of 1001 reported cases. Am Surg 1994; 60: 441-446

6 Rodríguez Hermosa JI, Codina Cazador A, Gironès Vilà J, Roig García J, Figa Francesch M, Acero Fernández D. Gallstone Ileus: results of analysis of a series of 40 patients. Gastroenterol Hepatol 2001; 24: 489-494

7 Lobo DN, Jobling JC, Balfour TW. Gallstone ileus: diagnostic 
pitfalls and therapeutic successes. J Clin Gastroenterol 2000; 30: 72-76

8 Everhart JE, Khare M, Hill M, Maurer KR. Prevalence and ethnic differences in gallbladder disease in the United States. Gastroenterology 1999; 117: 632-639

9 Berger MY, van der Velden JJ, Lijmer JG, de Kort H, Prins A, Bohnen AM. Abdominal symptoms: do they predict gallstones? A systematic review. Scand J Gastroenterol 2000; 35: 70-76

10 Newman HF, Northup JD, Rosenblum M, Abrams H. Complications of cholelithiasis. Am J Gastroenterol 1968; 50: $476-496$

11 Glenn F, Reed C, Grafe WR. Biliary enteric fistula. Surg Gynecol Obstet 1981; 153: 527-531

12 Kasahara Y, Umemura H, Shiraha S, Kuyama T, Sakata K, Kubota H. Gallstone ileus. Review of 112 patients in the Japanese literature. Am J Surg 1980; 140: 437-440

13 Balthazar EJ, Schechter LS. Air in gallbladder: a frequent finding in gallstone ileus. AJR Am J Roentgenol 1978; 131: 219-222

14 Lasson A, Lorén I, Nilsson A, Nirhov N, Nilsson P. Ultrasonography in gallstone ileus: a diagnostic challenge. Eur J Surg 1995; 161: 259-263

15 Lassandro F, Gagliardi N, Scuderi M, Pinto A, Gatta G, Mazzeo R. Gallstone ileus analysis of radiological findings in 27 patients. Eur J Radiol 2004; 50: 23-29

16 Yu CY, Lin CC, Shyu RY, Hsieh CB, Wu HS, Tyan YS, Hwang JI, Liou CH, Chang WC, Chen CY. Value of CT in the diagnosis and management of gallstone ileus. World J Gastroenterol 2005;

\section{1: $2142-2147$}

17 Agresta F, Bedin N. Gallstone ileus as a complication of acute cholecystitis. Laparoscopic diagnosis and treatment. Surg Endosc 2002; 16: 1637

18 Syme RG. Management of gallstone ileus. Can J Surg 1989; 32: 61-64

19 Zuegel N, Hehl A, Lindemann F, Witte J. Advantages of onestage repair in case of gallstone ileus. Hepatogastroenterology 1997; 44: 59-62

20 Tan YM, Wong WK, Ooi LL. A comparison of two surgical strategies for the emergency treatment of gallstone ileus. Singapore Med J 2004; 45: 69-72

21 Doko M, Zovak M, Kopljar M, Glavan E, Ljubicic N, Hochstädter H. Comparison of surgical treatments of gallstone ileus: preliminary report. World J Surg 2003; 27: 400-404

22 Franklin ME, Dorman JP, Schuessler WW. Laparoscopic treatment of gallstone ileus: a case report and review of the literature. J Laparoendosc Surg 1994; 4: 265-272

23 Dumonceau JM, Delhaye M, Devière J, Baize M, Cremer M. Endoscopic treatment of gastric outlet obstruction caused by a gallstone (Bouveret's syndrome) after extracorporeal shockwave lithotripsy. Endoscopy 1997; 29: 319-321

24 Meyenberger C, Michel C, Metzger U, Koelz HR. Gallstone ileus treated by extracorporeal shockwave lithotripsy. Gastrointest Endosc 1996; 43: 508-511

25 van Hillo M, van der Vliet JA, Wiggers T, Obertop H, Terpstra OT, Greep JM. Gallstone obstruction of the intestine: an analysis of ten patients and a review of the literature. Surgery 1987; 101: 273-276

S- Editor Liu Y L- Editor Wang XL E- Editor Chin GJ 